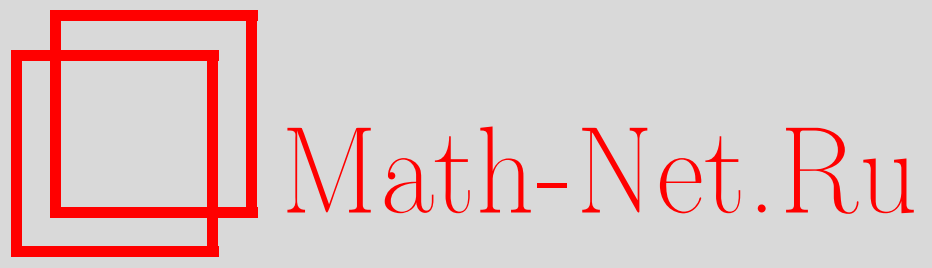

K. Куи, Т. Шибата, Стратегии, связанные с моментом инвестирования и размером вкладываемой суммы, в условиях асимметричной информации, Теория вероятн. и ее примен., 2016, том 61, выпуск 1, 165-173

DOI: https://doi.org/10.4213/tvp5048

Использование Общероссийского математического портала Math-Net.Ru подразумевает, что вы прочитали и согласны с пользовательским соглашением http://www . mathnet.ru/rus/agreement

Параметры загрузки:

IP: 54.209 .52 .79

26 апреля 2023 г., $16: 30: 48$

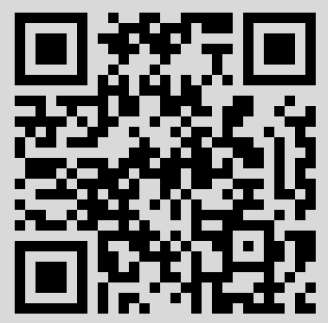


(c) 2016 г.

КУИ К.* Ш ШИБАТА Т.*

\title{
СТРАТЕГИИ, СВЯЗАННЫЕ С МОМЕНТОМ ИНВЕСТИРОВАНИЯ И РАЗМЕРОМ ВКЛАДЫВАЕМОЙ СУММЫ, В УСЛОВИЯХ АСИММЕТРИЧНОЙ ИНФОРМАЦИИ ${ }^{1)}$
}

\author{
(Перевод Жуленева С.В.)
}

\begin{abstract}
Мы обобщаем проблему асимметричной информации, включая в нее не только решение о моменте инвестирования, но также и стратегию определения размера инвестиций. Мы показываем, что момент инвестиции откладывается на больший срок при асимметричной, чем при полной информации, следствием чего является уменьшение стоимости акций. Однако, чтобы уменьшить эту неэффективность, инвестиционные вложения выбираются большими при асимметричной, чем при полной информации. Таким образом, при асимметричной информации имеет место компромисс между эффективностями от момента инвестиции и от ее величины.
\end{abstract}

Ключевые слова и фразы: инвестиции в условиях неопределенности, реальные опционы, информационная рента, частная информация.

1. Введение. Модель реальных опционов стала стандартным подходом к корпоративным инвестиционным решениям. В стандартной модели реальных опционов предполагается, что фирма управляется собственниками ${ }^{2)}$. Однако в последнее время собственники большинства корпораций поручают управление менеджерам, чтобы иметь возможность использовать их профессиональное мастерство и опыт. А в этой ситуации вероятно, что асимметричная информация существует, поскольку менеджеры обладают и частной информацией. Таким образом, асимметричная информация ведет к конфликтам в организации между собственниками и менеджерами.

В последнее время асимметричная информация между собственниками и менеджерами рассматривалась в нескольких исследованиях, в которых она встраивалась в модель реальных опционов. Гренадье и Ванг [2] разработали модель реальных опционов при наличии асимметричной информации между собственниками и управляющими ${ }^{3)}$. В подобной ситуации собственники должны составлять контракт, точно описывающий механизм, который бы заставлял менеджеров показывать частную информацию правдиво. Выбираемый в связи с этим момент инвестиции откладывается по сравнению с соответствующим моментом при полной (симметричной) информации, что ведет к уменьшению цены акций (стоимости собственников). Несмотря на то, что эти стратегии оказываются хуже оптимальных, они уменьшают потери собственников, возникающие от асимметричной информации. Без какого-либо механизма, заставляющего менеджеров показывать частную информацию правдиво, собственники страдали бы и от других неприятностей.

* Graduate School of Social Sciences, Tokyo Metropolitan University, Tokyo, Japan; e-mail: xcuiaa@gmail.com, tshibata@tmu.ac.jp

1) Исследование выполнено при поддержке Азиатского фонда по человеческим ресурсам Токийского столичного правительства, JSPS KAK-ENHI (гранты № 26242028, 26285071) и Фонда телекоммуникационных достижений.

2) Великолепный обзор по подходу, основанному на стандартных реальных опционах, заинтересованный читатель может найти в [1].

3) Обобщение идей работы [2] см. в [4] и [5]. 
Насколько нам известно, исследования стратегии размеров инвестиций в модели реальных опционов при асимметричной информации не проводились. А значит, остаются без ответа следующие вопросы. Как асимметричная информация влияет на размер инвестиций? Как асимметричная информация сказывается на связи между стратегиями выбора момента инвестиции и ее размера? Именно поэтому в настоящей работе мы рассматриваем взаимовлияние между стратегиями по выбору момента инвестиции и по выбору ее размера. В частности, мы обобщаем модель ГренадьеВанга [2], встраивая в нее решения по размеру инвестиций.

Мы показываем, что момент инвестирования откладывается на больший срок при асимметричной информации, чем при полной информации, следствием чего является уменьшение стоимости акций. Однако, чтобы уменьшить эту неэффективность, инвестиционные вложения можно сделать большими при асимметричной, чем при полной информации. Из этих результатов следует, что при асимметричной информации эффективность момента инвестиции меньше, а эффективность выбора инвестиционных сумм выше. Таким образом, при асимметричной информации имеет место компромисс между эффективностями от момента инвестиции и от инвестируемой суммы.

Статья организована следующим образом. Суть нашей модели описывается в разделе 2. При этом мы сочли полезным, прежде чем анализировать проблему с асимметричной информацией, рассмотреть в качестве исходного пункта проблему с полной информацией. В разделе 3 приводится решение проблемы асимметричной информации, затем на конкретных численных примерах обсуждаются свойства этого решения. Заключительные замечания приведены в разделе 4. В приложении (раздел 5) содержатся детали доказательств.

2. Описание моделей. Этот раздел мы начинаем с описания сути (структуры) модели. Затем формулируется проблема асимметричной информации. В заключение мы для сравнения приводим решение проблемы полной информации.

2.1. Постановка задачи. Собственник фирмы имеет право инвестировать в один-единственный проект. Мы предполагаем, что собственник (принципал) делегирует право принимать решения менеджеру (агенту). Везде в нашем анализе мы считаем, что собственник и менеджер риск-нейтральны и нацелены на максимизацию ожидаемых выплат.

Если инвестиционный опцион исполняется в момент $t$, то по наступлении этого момента фирма получает денежную сумму $\delta X_{t}$, где $\delta \geqslant 1$ и $X_{t}$ суть проекта и цена соответственно. Цена $X_{t}$ описывается процессом геометрического броуновского движения:

$$
d X_{t}=\mu X_{t} d t+\sigma X_{t} d z_{t}, \quad X_{0}=x,
$$

где $z_{t}$ - стандартное броуновское движение, а $\mu>0$ и $\sigma>0$ - некоторые фиксированные числа. Для обеспечения сходимости предполагается, что $r>\mu$, где $r>0-$ постоянная процентная ставка ${ }^{4)}$.

Мы предполагаем, что одномоментная фиксированная стоимость для начала реализации проекта есть $F(\delta):=I+c(\delta)$, где $F^{\prime}(\delta)>0, F^{\prime \prime}(\delta)>0$ при любом $\delta$. Отметим, что в момент вложения денег величина $\delta \geqslant 1$ выбирается эндогенно, чтобы максимизировать прибыль собственника ${ }^{5)}$. Чтобы гарантировать существование единственного решения $\delta$, предполагается, что $\delta F^{\prime}(\delta) / F(\delta)$ возрастает по $\delta$ (подробности см. в разделе 5). Это условие означает, что эластичность функции стоимости возрастает вместе с $\delta$. Кроме того, мы считаем, что $I \in\left\{I_{1}, I_{2}\right\}$, где $I_{2}>I_{1}>0$. Мы обозначаем $\Delta I:=I_{2}-I_{1}>0$, предполагая, что $I_{1}$ представляет собой расходы по «нижней

4) Условие $r>\mu$ нужно для того, чтобы стоимость фирмы была конечной.

5) В стандартной модели Дикзита и Пиндика [1] величина $\delta$ равна 1 и предполагается экзогенной. 
стоимости», а $I_{2}$ - расходы по «верхней стоимости». Вероятность выбора $I_{1}$ равна $\mathbb{P}\left(I_{1}\right)=q>0$, экзогенной переменной.

Мы предполагаем, что за денежным потоком $\delta X_{t}$ наблюдают оба - и собственник, и менеджер. Однако за $I$ частным образом наблюдает лишь менеджер ${ }^{6)}$. Сразу после заключения контракта с собственником в нулевой момент менеджер уточняет, принадлежат денежные расходы «нижней стоимости» или «верхней стоимости». С другой стороны, собственник не может наблюдать за истинным значением величины $I$, и, следовательно, он должен заставить менеджера использовать частную информацию честно в момент, когда менеджер производит инвестиции. В противном случае собственник понесет излишние потери. Предположим, например, что менеджер наблюдает равенство $I=I_{1}$, т.е. понимает, что реальное значение величины $I$ есть $I_{1}$. Однако он докладывает собственнику, что $I=I_{2}$, оставляя разницу $\Delta I>0$ себе. Чтобы избежать подобных нарушений, собственник должен введением стимулов поощрить менеджера докладывать истинное значение.

Полезно ввести оператор приведенного значения (т.е. текущей стоимости) одного доллара, полученного в момент первого достижения величиной $X_{t}$ порога $x_{i}$. Пусть $x_{i}=x\left(I_{i}\right)$ и $\delta_{i}=\delta\left(I_{i}\right)$ обозначают порог инвестирования и саму вносимую денежную сумму для $I=I_{i}(i \in\{1,2\})$ соответственно. Пусть $\tau_{i}$ обозначает момент остановки, в который инвестиции производятся, как только величина $X_{t}$ достигает порога $x_{i}$, т.е. $\tau_{i}:=\inf \left\{t>0 \mid X_{t} \geqslant x_{i}\right\}$. Пусть $\mathbb{E}^{x}\left[e^{-r \tau_{i}}\right]$ - оператор дисконтированного приведенного значения, где $\mathbb{E}^{x}[\cdot]$ обозначает оператор условного математического ожидания при условии, что $X_{0}=x$. Используя стандартную аргументацию, получаем, что

$$
\mathbb{E}^{x}\left[e^{-r \tau_{i}}\right]=\left(\frac{x}{x_{i}}\right)^{\beta}, \quad i \in\{1,2\}
$$

где $x<x_{i}$, а $\beta=1 / 2-\mu / \sigma^{2}+\sqrt{\left(\mu / \sigma^{2}-1 / 2\right)^{2}+2 r / \sigma^{2}}>1$.

2.2. Модель с асимметричной информацией. В этом пункте мы формулируем задачу максимизации для собственника при условии асимметричной информации. Как объяснялось ранее, при условии асимметричной информации собственник должен побудить менеджера показывать частную информацию правдиво.

В этом исследовании предполагается, что собственник в нулевой момент составляет контракт, обязывающий его простимулировать менеджера в момент проведения инвестиций. При этом повторное обсуждение условий не допускается. Поскольку принятое обязательство может привести к апостериорной неэффективности момента инвестиций, то оно увеличивает априорную стоимость опциона собственника. Предполагается, что для того, чтобы мотивировать менеджера показывать частную информацию, собственник стимулирует его бонусом $w_{i}=w\left(I_{i}\right)$ в момент инвестиции. Поскольку в положении равновесия менеджер в качестве частной информации показывает истинное $I_{i}$, мы не делаем различия между показанным $\tilde{I}_{i}$ и реальным $I_{i}$.

Таким образом, контракт в задаче с асимметричной информацией моделируется в виде механизма $\mathscr{M}^{* *}=\left(x_{i}, \delta_{i}, w_{i}\right)$, где $i \in\{1,2\}$. Верхний индекс ${ }^{* *}$ относится к задаче с асимметричной информацией. Заметим, что контракт $\mathscr{M}^{* *}$ состоит из порога $x_{i}$, величины $\delta_{i}$ и стимулирующего бонуса $w_{i}$ при любом $i$.

Поэтому задача с асимметричной информацией сводится к максимизации стоимости опциона собственника на множестве возможных значений механизма $\mathscr{M}^{* *}$, т.е. к

6) Поскольку величина $\delta X_{t}$ является наблюдаемой, то менеджер обладает информацией о $c(\delta)$. Предположение, что за частью стоимости проекта частным образом наблюдает лишь одна сторона (здесь менеджер) и не следит другая (здесь собственник), свойственно литературе по асимметричной информации. Превосходный обзор по анализу ситуаций с асимметричной информацией имеется в [3]. 
экстремальной задаче

$$
\max _{x_{1}, x_{2}, \delta_{1}, \delta_{2}, w_{1}, w_{2}} \sum_{i=1}^{2} \mathbb{P}\left(I_{i}\right)\left(\frac{x}{x_{i}}\right)^{\beta}\left\{\delta_{i} x_{i}-I_{i}-w_{i}-c\left(\delta_{i}\right)\right\}
$$

с условиями

$$
\begin{gathered}
\left(\frac{x}{x_{1}}\right)^{\beta} w_{1} \geqslant\left(\frac{x}{x_{2}}\right)^{\beta}\left(w_{2}+\Delta I\right), \\
\left(\frac{x}{x_{2}}\right)^{\beta} w_{2} \geqslant\left(\frac{x}{x_{1}}\right)^{\beta}\left(w_{1}-\Delta I\right), \\
w_{i} \geqslant 0, \quad i \in\{1,2\} .
\end{gathered}
$$

Целевая функция (3) в этой задаче и представляет априорную стоимость опциона собственника. Отметим, что эта проблема при фиксированном $\delta$ (например, при $\delta=$ 1) совпадает с проблемой Гренадье-Ванга [2].

Ограничения (4) и (5) являются апостериорными ограничениями по совместимости стимулов для менеджера при состояниях $I_{1}$ и $I_{2}$ соответственно. Рассмотрим, например, ограничение (4). В состоянии $I_{1}$ выплата менеджеру равна $\left(x / x_{1}\right)^{\beta} w_{1}$, если менеджер говорит правду, и $\left(x / x_{2}\right)^{\beta}\left(w_{2}+\Delta I\right)$, если менеджер вместо этого заявляет о состоянии $I_{2}$. Таким образом, менеджер говорит правду, если (4) выполнено. Ограничение (5) обосновывается аналогично. Ограничения (6) представляют апостериорную ограниченность обязательств.

Прежде чем анализировать проблему с асимметричной информацией, мы кратко напомним постановку задачи с полной (симметричной) информацией.

2.3. Задача с полной информацией. В этом пункте мы рассмотрим экстремальную задачу, в которой собственник наблюдает реальное значение $I$. Эта проблема эквивалентна проблеме, в которой менеджеру не поручается принимать решение об инвестициях, поскольку в обладании информацией у него нет преимуществ. Поэтому $w_{i}=0$ при любом $i \in\{1,2\}$. Таким образом, контракт $\mathscr{M}^{*}$ в случае задачи с полной информацией моделируется в виде $\mathscr{M}^{*}=\left(x_{i}, \delta_{i}\right), \quad i \in\{1,2\}$. Отнесем верхний индекс ${ }^{*}$ к проблеме с полной (симметричной) информачией. Экстремальную задачу для собственника определим следующим образом:

$$
\max _{x_{1}, x_{2}, \delta_{1}, \delta_{2}} q V\left(x, x_{1}, \delta_{1} ; I_{1}\right)+(1-q) V\left(x, x_{2}, \delta_{2} ; I_{2}\right),
$$

где $x<x_{i}$ при любом $i(i \in\{1,2\})$, а

$$
V\left(x, x_{i}, \delta_{i} ; I_{i}\right):=\left(\frac{x}{x_{i}}\right)^{\beta}\left\{\delta_{i} x_{i}-I_{i}-c\left(\delta_{i}\right)\right\} .
$$

Имеем следующий результат (доказательство отнесено в приложение, см. п. 5.1).

Теорема А. В случае задачи с полной информачией величина $\delta_{i}^{*}$ является при любом $i \in\{1,2\}$ решением следуюшего уравнения:

$$
\frac{\beta}{\beta-1}=\frac{c^{\prime}\left(\delta_{i}^{*}\right) \delta_{i}^{*}}{I_{i}+c\left(\delta_{i}^{*}\right)}
$$

а $x_{i}^{*}$ задается выражением

$$
x_{i}^{*}=\frac{\beta}{\beta-1} \frac{I_{i}+c\left(\delta_{i}^{*}\right)}{\delta_{i}^{*}} .
$$

Стоимость собственника определяется равенством

$$
o^{*}(x)=q V\left(x, x_{1}^{*}, \delta_{1}^{*} ; I_{1}\right)+(1-q) V\left(x, x_{2}^{*}, \delta_{2}^{*} ; I_{2}\right) .
$$

Напомним, что правая часть равенства $(9), c^{\prime}\left(\delta_{i}\right) \delta_{i} /\left(I_{i}+c\left(\delta_{i}\right)\right)=F^{\prime}\left(\delta_{i}\right) \delta_{i} / F\left(\delta_{i}\right)$, возрастает вместе с $\delta_{i}$. Поэтому существует единственное решение $\delta_{i}^{*}$. Результаты теоремы 1 мы используем как отправную точку. 
3. Решение задачи с асимметричной информацией. В этом пункте приводится решение описанной выше проблемы с асимметричной информацией. Затем анализируются некоторые свойства этого решения.

Хотя в нашей проблеме оптимизации ограничения состоят из четырех неравенств, ее можно упростить за следующие два шага. Во-первых, в отличие от менеджера в состоянии $I_{1}$, менеджер в состоянии $I_{2}$ не имеет стимула говорить неправду. Это происходит потому, что менеджер в состоянии $I_{2}$ понесет потери от лживого объявления. Таким образом, условие (5) выполняется автоматически и потому значение $w_{2}^{* *}=0$ оптимально. Во-вторых, предположим, что в (4) выполняется строгое неравенство. Тогда стоимость собственника увеличивается при уменьшении $w_{1}$. Таким образом, (4) приводит к тому, что $w_{1}^{* *}=\left(x_{1} / x_{2}\right)^{\beta} \Delta I$.

В результате упрощенная проблема оптимизации принимает вид

$$
\max _{x_{1}, x_{2}, \delta_{1}, \delta_{2}} q V\left(x, x_{1}, \delta_{1} ; I_{1}\right)+(1-q) V\left(x, x_{2}, \delta_{2} ; I_{2}+\phi \Delta I\right),
$$

где $\phi:=q /(1-q)>0$, а $x<x_{i}$ при любом $i \in\{1,2\}$. Справедлив следующий результат (доказательство см. в п. 5.1).

Теорема В. Решением проблемь асимметричной информации при $I=I_{1}$ являются $x_{1}^{* *}=x_{1}^{*}, \delta_{1}^{* *}=\delta_{1}^{*}, w_{1}^{* *}=\left(x_{1}^{*} / x_{2}^{* *}\right)^{\beta} \Delta I$. Если же $I=I_{2}$, то $\delta_{2}^{* *}$ является решением уравнения

$$
\frac{\beta}{\beta-1}=\frac{c^{\prime}\left(\delta_{2}^{* *}\right) \delta_{2}^{* *}}{I_{2}+\phi \Delta I+c\left(\delta_{2}^{* *}\right)},
$$

$x_{2}^{* *}$ определяется формулой

$$
x_{2}^{* *}=\frac{\beta}{\beta-1} \frac{I_{2}+\phi \Delta I+c\left(\delta_{2}^{* *}\right)}{\delta_{2}^{* *}},
$$

a $w_{2}^{* *}=0$. Стоимость собственника и стоимость менеджера суть соответственно

$$
o^{* *}(x)=q V\left(x, x_{1}^{*}, \delta_{1}^{*} ; I_{1}\right)+(1-q) V\left(x, x_{2}^{* *}, \delta_{2}^{* *} ; I_{2}+\phi \Delta I\right)
$$

$u$

$$
m^{* *}(x)=q\left(\frac{x}{x_{2}^{* *}}\right)^{\beta} \Delta I .
$$

В связи с теоремой 2 сделаем четыре важных замечания. Во-первых, решение $\delta_{2}^{* *}$ единственно, поскольку правая часть в $(13), c^{\prime}\left(\delta_{2}\right) \delta_{2} /\left(I_{2}+\phi \Delta I+c\left(\delta_{2}\right)\right)$, возрастает по $\delta_{2}{ }^{7)}$. Во-вторых, имеем соотношения $x_{1}^{* *}=x_{1}^{*}, \delta_{1}^{* *}=\delta_{1}^{*}, x_{2}^{* *} \neq x_{2}^{*}, \delta_{2}^{* *} \neq \delta_{2}^{*}-$ ведь для собственника менее обременительно (дорого) сделать $\left(x_{2}^{* *}, \delta_{2}^{* *}\right)$ отличным от $\left(x_{2}^{*}, \delta_{2}^{*}\right)$, чем сделать $\left(x_{1}^{* *}, \delta_{1}^{* *}\right)$ отличным от $\left(x_{1}^{*}, \delta_{1}^{*}\right)$. В-третьих, у нас $w_{1}^{* *} \in(0, \Delta I)$ и $w_{2}^{* *}=0$. Поскольку $\Delta I>0$, то эту разность можно рассматривать как информационную ренту для менеджера в состоянии $I_{1}$, и потому собственник отдает менеджеру в состоянии $I_{1}$ долю этой ренты за использование частной информации. Наконец, у нас $o^{* *}(x)<o^{*}(x)$, что вытекает из неравенства $V\left(x, x_{2}^{* *}, \delta_{2}^{* *} ; I_{2}+\phi \Delta I\right)<V\left(x, x_{2}^{*}, \delta_{2}^{*} ; I_{2}\right)$. Таким образом, неэффективность является следствием информационной ренты величины $\phi \Delta I>0$.

Обсудим свойства решения проблемы асимметричной информации, используя эндогенную величину инвестиций. Справедлив следующий результат (доказательство см. в п. 5.3).

Теорема С. Справедливо неравенство $\delta_{2}^{* *}>\delta_{2}^{*}$. При оптимальной величине инвестииий $\delta_{2}^{* *}$ мьи имеем $x_{2}^{* *}>x_{2}^{*} u V\left(x, x_{2}^{* *}, \delta_{2}^{* *} ; I_{2}+\phi \Delta I\right)<V\left(x, x_{2}^{*}, \delta_{2}^{*} ; I_{2}\right)$. Кроме того, увеличение разности $\Delta I$ приводит $\kappa$ увеличению $x_{2}^{* *}, u \delta_{2}^{* *}$ и уменьшению $V\left(x, x_{2}^{* *}, \delta_{2}^{* *} ; I_{2}+\phi \Delta I\right)$.

7) Это вытекает из предположения, что $\delta F^{\prime}(\delta) / F(\delta)$ возрастает по $\delta$. 
Теорема 3 утверждает, что существует компромисс между эффективностями стратегий выбора момента инвестиции и величины ее суммы. Неравенство $x_{2}^{* *}>x_{2}^{*}$ означает, что при асимметричной информации фирма будет осуществлять инвестиции позже, чем при полной. А из неравенства $\delta_{2}^{* *}>\delta_{2}^{*}$ следует, что фирма предпримет инвестииии в больших объемах при асимметричной информаиии, чем при полной. Таким образом, при асимметричной информации, поскольку фирма страдает от потерь из-за отложенных инвестиций, она для компенсации этих потерь увеличивает вкладываемые суммы. Иными словами, существует компромисс между эффективностями стратегий выбора момента инвестиции и величины ее суммы. Эти свойства схожи с установленными в [6], где имеется компромисс между эффективностями от выбора момента инвестиции и от усилий менеджеров.

В подтверждение свойств решений рассмотрим численные примеры. Для этого предположим, что величина инвестируемых сумм определяется равенством

$$
c\left(\delta_{i}\right)=\delta_{i}^{2}, \quad i \in\{1,2\},
$$

а основные параметры имеют значения следующие значения: $r=0.09, \mu=0.02$, $\sigma=0.15, I_{1}=5, I_{2}=10$, а $x=5$.

В табл. 1 приведены численные результаты. В частности $x_{1}^{*}=x_{1}^{* *}=10.2864$, $x_{2}^{*}=14.5472$, а $x_{2}^{* *}=17.8165$. Тем самым подтверждается неравенство $x_{2}^{* *}>x_{2}^{*}$. Таким образом, даже при эндогенной инвестируемой сумме приходим к тому же самому результату $x_{2}^{* *}>x_{2}^{*}$, что и в [2]. Кроме того, мы получаем, что $\delta_{1}^{*}=\delta_{1}^{* *}=5.1432$, $\delta_{2}^{*}=7.2738$, а $\delta_{2}^{* *}=8.9072$. Наиболее важным результатом является неравенство $\delta_{2}^{* *}>\delta_{2}^{*}$. Помимо этого отметим, что $x_{2}^{* *}=c^{\prime}\left(\delta_{2}^{* *}\right)$, а $x_{2}^{*}=c^{\prime}\left(\delta_{2}^{*}\right)^{8)}$. Из этих равенств следует, что $x_{2}^{* *} / x_{2}^{*}=\delta_{2}^{* *} / \delta_{2}^{*}>1$ при условии, что функция стоимости определяется равенством $c\left(\delta_{i}\right)=\delta_{i}^{2}$. Более того, мы имеем $V_{1}^{*}=3.8212, V_{2}^{*}=3.0810, V_{2}^{* *}=2.8013$, где $V_{i}^{*}:=V\left(x, x_{i}^{*}, \delta_{i}^{*} ; I_{i}\right)$ и $\left.V_{2}^{* *}:=V\left(x, x_{2}^{* *}, \delta_{2}^{* *} ; I_{2}+\phi\right) \Delta I\right)$. Таким образом, асимметричная информация приводит к увеличению инвестиционного порога и размещаемых сумм, а также к уменьшению стоимости собственника.

Таблица 1. Оптимальные решения и стоимости

\begin{tabular}{|l|c|c|}
\hline & Симметричная (полная) информация $(*)$ & Асимметричная информация $(* *)$ \\
\hline \hline$x_{1}$ & 10.2864 & 10.2864 \\
$\delta_{1}$ & 5.1432 & 5.1432 \\
\hline$x_{2}$ & 14.5472 & 17.8165 \\
$\delta_{2}$ & 7.2738 & 8.9072 \\
\hline$V_{1}$ & 3.8212 & 3.8212 \\
$V_{2}$ & 3.0810 & 2.8013 \\
\hline
\end{tabular}

Напомним, что наша модель преврашается в модель Гренадье-Ванга [2], если размешаемая сумма $\delta$ задается экзогенно. Рис. 1 демонстрируют эффект от эндогенно инвестируемой денежной суммы $\delta$. На рис. $1($ a) указаны инвестиционные пороги. При пороге асимметричной информации, равном $x_{2}^{* *}$, мы выбираем $\delta_{2}=5.1432$ и $\delta_{2}=$ 8.9072 в качестве исходной точки, что соответствует оптимальным величинам $\delta_{2}^{* *}$ при $\Delta I=0$ и $\Delta I=5$. Мы имеем $x_{2}^{* *}\left(\delta_{2}=5.1432\right) \leqslant x_{2}^{* *}\left(\delta_{2}^{* *}\right) \leqslant x_{2}^{* *}\left(\delta_{2}=8.9072\right)^{9)}$, где $x_{2}^{* *}\left(\delta_{2}\right)$ есть инвестиционный порог при фиксированном $\delta_{2}$. При любом $\delta_{2}\left(\delta_{2} \in\right.$ $\left.\left\{5.1432, \delta_{2}^{* *}, 8.9072\right\}\right)$ величина $x_{2}^{* *}\left(\delta_{2}\right)$ возрастает по $\Delta I$.

8) Преобразование равенств (13) и (14) приводит к первому равенству. Второе равенство вытекает из (9) и (10).

9) Аналогично, при пороге полной информации, равном $x_{2}^{*}$, мы полагаем $\delta_{2}=5.1432$ и $\delta_{2}=7.2738$ при $\Delta I=0$ и $\Delta I=5$ соответственно. И мы имеем $x_{2}^{*}\left(\delta_{2}=5.1432\right) \leqslant$ $x_{2}^{*}\left(\delta_{2}^{*}\right) \leqslant x_{2}^{*}\left(\delta_{2}=7.2738\right)$. 


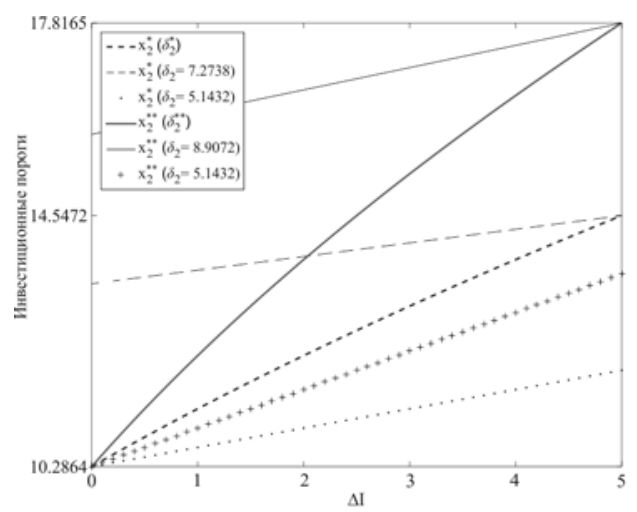

(a)

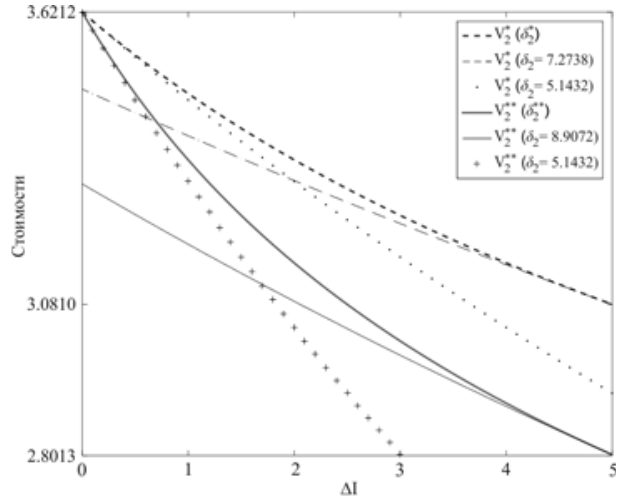

(b)

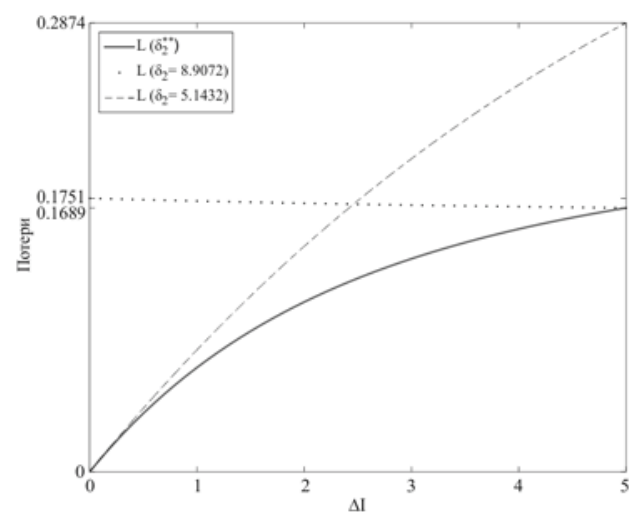

(c)

Рис. 1. Сравнения с моделью Гренадье-Ванга.

На рис. 1(b) изображены $V_{2}^{*}\left(\delta_{2}\right)$ и $V_{2}^{* *}\left(\delta_{2}\right)$, где $V_{2}^{*}(y)=V\left(x, x_{2}^{*}, y ; I_{2}\right)$ и $V_{2}^{* *}(y)=$ $V\left(x, x_{2}^{* *}, y ; I_{2}+\phi \Delta I\right)$. Отсюда сразу следует, что $V_{2}^{* *}\left(\delta_{2}^{* *}\right) \geqslant V_{2}^{* *}\left(\delta_{2}\right)$ при любом $\delta_{2}$ (например, при $\left.\delta_{2} \in\{5.1432,8.9072\}\right)$. Величина $V_{2}^{* *}\left(\delta_{2}\right)$ убывает по $\Delta I$ при любом $\delta_{2}$.

Для измерения неэффективности, возникающей от асимметричной информации, мы определяем функцию потерь $L:=o^{*}(x)-o^{* *}(x)-m^{* *}(x)$ в виде

$$
L\left(\delta_{2}\right)=(1-q)\left(V\left(x, x_{2}^{*}, \delta_{2} ; I_{2}\right)-V\left(x, x_{2}^{* *}, \delta_{2} ; I_{2}\right)\right) \geqslant 0 .
$$

На рис. $1\left(\right.$ с) изображена зависимость $L\left(\delta_{2}\right)$ от $\Delta I$. Ясно, что $L\left(\delta_{2}^{* *}\right) \leqslant L\left(\delta_{2}\right)$ при любом $\delta_{2}$ (например, при $\left.\delta_{2} \in\{5.1432,8.9072\}\right)$. Еще интересно, что $L\left(\delta_{2}\right)$ убывает по $\Delta I$ при $\delta_{2}=8.9072$, в то время как $L\left(\delta_{2}\right)$ возрастает по $\Delta I$ при $\delta_{2}=5.1432$. При оптимальном $\delta_{2}^{* *}$ величина $L\left(\delta_{2}\right)$ возрастает по $\Delta I$.

4. Заключительные замечания. В нашей работе проанализированы стратегии выбора момента и величины инвестируемых сумм в случае асимметричной информации собственника и менеджера, а именно в случае, когда у менеджера есть информационное преимущество. Установлено, что при асимметричной информации момент инвестирования смещается вперед по времени по сравнению со случаем полной информации, в то время как инвестируемая сумма при асимметричной информации становится больше, чем при полной. Мы заключаем, что при асимметричной информации эффективность от инвестируемых сумм выше, а эффективность от момента инвестиции при таком же сравнении ниже, чем при полной информации. 


\section{5. Приложение.}

5.1. Доказательство теорем 1 и 2. Поскольку доказательства этих теорем одинаковы, мы ограничимся доказательством теоремы 2.

Мы приведем решение для случая $I=I_{2}$. А чтобы упростить обозначения, опустим верхний индекс ${ }^{* *}$ и будем писать просто $x_{2}$ и $\delta_{2}$. Дифференцируя $V\left(x, x_{2}, \delta_{2} ; I_{2}+\right.$ $\phi \Delta I)$ по $x_{2}$ и $\delta_{2}$, получим

$$
\begin{aligned}
\frac{\partial V}{\partial x_{2}} & =\left(\frac{x}{x_{2}}\right)^{\beta}\left(\frac{-\beta}{x_{2}}\left\{\delta_{2} x_{2}-I_{2}-\phi \Delta I-c\left(\delta_{2}\right)\right\}+\delta_{2}\right)=0, \\
\frac{\partial V}{\partial \delta_{2}} & =\left(\frac{x}{x_{2}}\right)^{\beta}\left\{x_{2}-c^{\prime}\left(\delta_{2}\right)\right\}=0
\end{aligned}
$$

соответственно. Условие второго порядка выполнено, поскольку

$$
M_{11}<0 \quad \text { и }|M|>0,
$$

где матрица $M$ определяется в виде

$$
M=\left(\begin{array}{cc}
\frac{\partial^{2} V}{\partial x_{2}^{2}} & \frac{\partial^{2} V}{\partial \delta_{2} \partial x_{2}} \\
\frac{\partial^{2} V}{\partial x_{2} \partial \delta_{2}} & \frac{\partial^{2} V}{\partial \delta_{2}^{2}}
\end{array}\right)=\theta\left(\begin{array}{cc}
\frac{\delta_{2}}{x_{2}}(1-\beta) & 1 \\
1 & -c^{\prime \prime}\left(\delta_{2}\right)
\end{array}\right)
$$

и $\theta:=\left(x / x_{2}\right)^{\beta} \in(0,1)$. Заметим, что $M_{11}<0$, поскольку $\beta>1$. Для вывода $(22)$ использованы соотношения (19) и (20). Преобразуя (19) и (20), получаем для $\delta_{2}^{* *}$ и $x_{2}^{* *}$ формулы (13) и (14) соответственно.

Рассмотрим условие, из которого вытекает неравенство $|M|>0$. Заметим, что $x_{2}=c^{\prime}\left(\delta_{2}\right)$ в $(20)$. Таким образом, если неравенство $\delta_{2} c^{\prime \prime}\left(\delta_{2}\right) / c^{\prime}\left(\delta_{2}\right)>(1-\beta)^{-1}$ выполнено, то мы и получим $|M|>0$. Кроме того, напомним, что в силу (13) единственное решение $\delta_{2}$ существует при условии, что функция $\delta_{2} c^{\prime}\left(\delta_{2}\right) /\left(I_{2}+\phi \Delta I+c\left(\delta_{2}\right)\right)$ возрастает по $\delta_{2}$. Дифференцируя эту функцию по $\delta_{2}$, получаем:

$$
\begin{aligned}
\left(\frac{\delta_{2} c^{\prime}\left(\delta_{2}\right)}{I_{2}+\phi \Delta I+c\left(\delta_{2}\right)}\right)^{\prime} & =\frac{1}{I_{2}+\phi \Delta I+c\left(\delta_{2}\right)}\left(\delta_{2} c^{\prime \prime}\left(\delta_{2}\right)+c^{\prime}\left(\delta_{2}\right)-\frac{\delta_{2} c^{\prime}\left(\delta_{2}\right)}{I_{2}+\phi \Delta I+c\left(\delta_{2}\right)} c^{\prime}\left(\delta_{2}\right)\right), \\
& =\frac{1}{I_{2}+\phi \Delta I+c(\delta)}\left(\delta c^{\prime \prime}(\delta)-(\beta-1)^{-1} c^{\prime}(\delta)\right)
\end{aligned}
$$

(при выводе последнего равенства использовано представление (13)). Таким образом, неравенство $\delta_{2} c^{\prime \prime}\left(\delta_{2}\right) / c^{\prime}\left(\delta_{2}\right)>(\beta-1)^{-1}$ влечет положительность $(23)$. В результате получаем, что условие $|M|>0$ эквивалентно положительности (23).

По условию в нашей модели функция $\delta F^{\prime}(\delta) / F(\delta)$, где $F(\delta):=I+c(\delta)$, возрастает по $\delta$, а отсюда следует, что $\delta F^{\prime}(\delta) /(F(\delta)+\phi \Delta I)$ возрастает по $\delta$.

Предположим, например, что $c(\delta)=\delta^{2}$, как в (17). Поскольку $\delta c^{\prime \prime}(\delta) / c^{\prime}(\delta)=1$, то условие, которое обеспечит неравенство $|M|>0$, сводится к неравенству $\beta>2$, откуда вытекает, что $\lim _{\delta \rightarrow \infty} \delta c^{\prime}(\delta) /(I+c(\delta))>\beta /(\beta-1)$. При основных параметрах (т.е. $r=0.09, \mu=0.02 \sigma=0.15$ ) имеем $\beta=2.4661$.

5.2. Доказательство теоремы 3. Покажем сначала, что $d x_{2}^{* *} / d \Delta I>0$ и $d \delta_{2}^{* *} / d \Delta I>0$. Дифференцирование равенств (19) и (20) приводит к соотношению $M y=p$, где $M$ имеем вид (22), а $y$ и $p$ определяются формулами

$$
y=\left(\begin{array}{c}
d x_{2}^{* *} \\
d \delta_{2}^{* *}
\end{array}\right), \quad p=\theta\left(\begin{array}{c}
-\beta \phi d \Delta I\left(x_{2}^{* *}\right)^{-1} \\
0
\end{array}\right) .
$$

Решая систему относительно $y$, получаем

$$
\frac{d x_{2}^{* *}}{d \Delta I}=\frac{\theta}{|M|} \frac{\beta}{x_{2}^{* *}} \phi c^{\prime \prime}\left(\delta_{2}^{* *}\right)>0, \quad \frac{d \delta_{2}^{* *}}{d \Delta I}=\frac{\theta}{|M|} \frac{\beta}{x_{2}^{* *}} \phi>0,
$$


где мы использовали тот факт, что $|M|>0$ в силу (21).

Теперь докажем неравенство $d V / d \Delta I<0$. Дифференцируя $V\left(x, x_{2}^{* *}, \delta_{2}^{* *} ; I_{2}+\phi \Delta I\right)$ по $\Delta I$, получаем, что

$\frac{d V\left(x, x_{2}^{* *}, \delta_{2}^{* *} ; I_{2}+\phi \Delta I\right)}{d \Delta I}=\frac{\partial V}{\partial x_{2}^{* *}} \frac{d x_{2}^{* *}}{d \Delta I}+\frac{\partial V}{\partial \delta_{2}^{* *}} \frac{d \delta_{2}^{* *}}{d \Delta I}+\frac{\partial V}{\partial \Delta I}=\frac{\partial V}{\partial \Delta I}=-\left(\frac{x}{x_{2}^{* *}}\right)^{\beta} \phi<0$ где при выводе второго равенства использована так называемая охватывающая теорема.

Мы выражаем признательность анонимному рецензенту за полезные комментарии и советы.

\section{СПИСОК ЛИТЕРАТУРЫ}

1. Dixit A.K., Pindyck R.S. Investment under Uncertainty. Princeton: Princeton Univ. Press, 1994, 488 p.

2. Grenadier S. R., Wang N. Investment timing, agency, and information. - J. Financial Economics, 2005, v. 75, № 3, p. 493-533.

3. Laffont J. J., Martimort D. The Theory of Incentives: The Principal-Agent Model. Princeton: Princeton Univ. Press, 2002, 440 p.

4. Nishihara M., Shibata T. The agency problem between the owner and the manager in real investment: The bonus-audit relationship. - Oper. Res. Lett., 2008, v. 36, №3, p. 291-296.

5. Shibata T. Investment timing, asymmetric information, and audit structure: A real options framework. - J. Econom. Dynam. Control, 2009, v. 33, № 4, p. 903-921.

6. Shibata T., Nishihara M. Interaction between investment timing and management effort under asymmetric information: Costs and benefits of privatized firms. European J. Oper. Res., 2011, v. 215, № 3, p. 688-696.

Поступила в редакцию 7.XII.2014

Исправленный вариант 8.IX.2015

(C) 2016 г.

ПАВЛОВ И. В.* , ШАМРАЕВА В. В.*, ЦВЕТКОВА И.В.*

\section{О СУЩЕСТВОВАНИИ МАРТИНГАЛЬНЫХ МЕР, УДОВЛЕТВОРЯЮЩИХ ОСЛАБЛЕННОМУ УСЛОВИЮ НЕСОВПАДЕНИЯ БАРИЦЕНТРОВ, В СЛУЧАЕ СЧЕТНОГО ВЕРОЯТНОСТНОГО ПРОСТРАНСТВА ${ }^{1)}$}

Для статических $(B, S)$-рынков со счетным числом состояний изучается специальное интерполяционное свойство мартингальных мер, дающее возможность преобразовать неполные безарбитражные рынки в полные. Получены достаточные условия на параметры рынка, обеспечивающие существование таких мартингальных мер.

Ключевые слова и фразы: специальная хааровская фильтрация, $(B, S)$-рынок, безарбитражность, полнота, мартингальная мера, хааровская интерполяция мартингала, ослабленное условие несовпадения барицентров.

* Кафедра высшей математики, Ростовский государственный строительный университет, Ростов-на-Дону, Россия; e-mail: pavloviv2005@mail.ru

1) Работа выполнена при поддержке РФФИ (проекты 16-01-00184а и 16-07-00888а). 